\title{
A Benchmark TEAM Problem for Multi-Objective Pareto Optimization in Magnetics: the Time-Harmonic Regime
}

\author{
Paolo Di Barba ${ }^{1}$,Fabrizio Dughiero ${ }^{2}$, Michele Forzan ${ }^{2}$, David A. Lowther ${ }^{3}$, Fellow, IEEE, \\ Maria Evelina Mognaschi ${ }^{1}$, Elisabetta Sieni ${ }^{4}$, Jan K. Sykulski ${ }^{5}$, Fellow, IEEE \\ ${ }^{1}$ Dept of Electrical, Computer and Biomedical Engineering, University of Pavia, Pavia, Italy \\ ${ }^{2}$ Dept of Industrial Engineering, University of Padua, Padua, Italy \\ ${ }^{3}$ Dept of Electrical and Computer Engineering, McGill University, Montreal, Québec, Canada \\ ${ }^{4}$ Dip.toScienze e Tecnologie Applicate, University of Insubria, Varese, Italy \\ ${ }^{5}$ Electronics and Computer Science, University of Southampton, Southampton, UK \\ \{paolo.dibarba, eve.mognaschi\}@unipv.it, \{fabrizio.dughiero,michele.forzan\}@unipd.it, elisabetta.sieni@uninsubria.it

\begin{abstract}
The paper reformulates and generalizes the TEAM benchmark, originally proposed for multiobjective optimization of magnetic devices under DC conditions, now extending it to the AC regime. A solution is furnished which has enabled an extensive search and reliable estimation of the shape of the Pareto front. Field uniformity and losses are considered with reference to a class of power inductors. It is argued that the benchmark provides a challenging target for new algorithms, especially those involving numerical modelling based on finite element codes, where the number of objective function calls needs to be minimized for practical designs.
\end{abstract}

Index Terms - Multi-objective optimization, magnetic field synthesis, time-harmonic finite-element analysis, TEAM benchmark.

\section{INTRODUCTION}

$\mathrm{T}$ EAM problems, available from the website of the International Compumag Society [1], are well established as a reference in the community. In the area of optimization of electromagnetic devices there are two particular benchmarks known as No 22 SMES Optimization Benchmark and No 25 Optimization of Die Press Model. Both have been used extensively for testing new single-objective optimization algorithms. For some time there has been a need to create a benchmark which could ultimately be used for multi-objective problems, with particular emphasis on Pareto optimization. In this paper we put forward a simple - but important in practical applications - model for assessing the quality of magnetic field produced by a distributed winding. In [2] the first benchmark for magnetic design under DC conditions was proposed and solved, while in [3] a particular application in induction heating was considered. Here the original TEAM benchmark is enhanced and extended to the AC regime with a twofold aim: to incorporate additional and proximity losses and to investigate the dependence of the Pareto front on the supply frequency. To this end, the uniformity of the field is usually of prime interest but power loss is also an important issue, especially in the context of energy efficient designs. The available design space has been extensively searched to produce reliable non-dominated solutions for further consideration in a Pareto sense. In the paper, the well known NSGA-II algorithm [4] is compared with the Wind Driven

Manuscript received July 15, 2019; revised ??? ??, 2019 and ??? ??, 2019; accepted ??? ??, 2019. Date of publication ??? ??, 2020; date of current version ??? ??, 2019. Corresponding author: Maria Evelina Mognaschi (e-mail:eve.mognaschi@unipv.it).

Color versions of one or more of the figures in this paper are available online at http://ieeexplore.ieee.org.

Digital Obiect Identifier (inserted by IEEE).
Optimization WDO [5], the micro-Biogeography Inspired Multiobjective Optimization $\mu \mathrm{BiMO}[6]$ and the Migration Non-dominated Sorted Genetic Algorithm MNSGA-III, a modification of Migration-NSGA-II method [7]. Further optimization algorithms may therefore be tested against the proposed benchmark.

\section{THE FORWARD PROBLEM}

A multi-turn air-cored winding is considered, with the width of each turn $w_{1}=15 \mathrm{~mm}$ and the height $h=10 \mathrm{~mm}$, while the gap between the turns is in the order of a few mm. Hollow turns, exhibiting a $4 \mathrm{~mm}$ wide hole, are assumed to allow for water circulation. A possible application is related to the calibration procedure of magnetic probes.

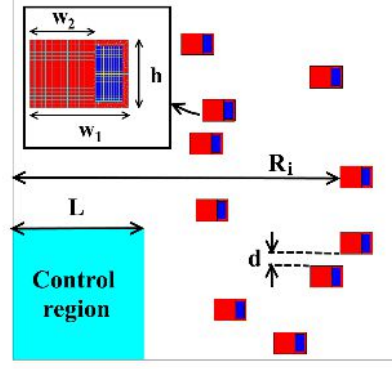

(a)

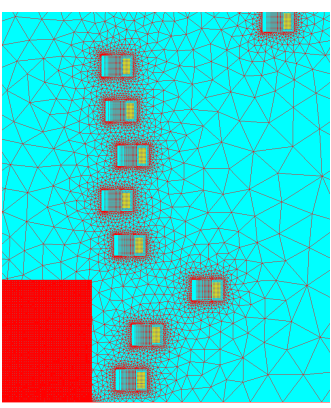

(b)
Fig. 1. Geometry, design variables and controlled region (a), mesh of the FE model (b).

The winding is composed of $n_{t}=20$ series-connected turns of the radii $R_{i}$ and is supplied by an AC current of 100 $\mathrm{A}_{\mathrm{rms}}$, the frequency of which can vary in the range [1-100] $\mathrm{kHz}$.

Due to a symmetric distribution, only half of the model is needed. For evaluating the field uniformity, a two- 
dimensional controlled region is considered: the magnitude of the magnetic field $H$ is sampled on a grid of $250 \times 250$ points, evenly spaced in a square region $\Omega$ with a side length $L$ equal to $60 \mathrm{~mm}$. The geometric model of the winding is shown in Fig. 1(a).

The problem, i.e. finding the magnetic field $\mathrm{H}$ given the current density $\mathrm{J}$ and the radii $\mathrm{R}$ distribution, was defined as an axisymmetric system. The magnetic analysis was then executed using time-harmonic conditions in Finite Element (FE) codes Flux [8] and MagNet [9].

A typical mesh is composed of nearly 18,000 second order triangular elements. Given that the penetration depth in copper at $100 \mathrm{kHz}$ is about a fifth of a millimeter, most of the conductor is not being used and the mesh density has to be appropriately set. In particular, in the copper turn, the maximum element size of the mesh is equal to an half of the penetration depth, given the frequency, i.e. $0.2 \mathrm{~mm}$ at $100 \mathrm{kHz}$.

A detail of the mesh is shown in Fig. 1b. In particular, the field problem was solved in terms of the complex magnetic vector potential, $\boldsymbol{A}$, as

$$
\nabla^{2} \dot{\boldsymbol{A}}-j \omega \mu \rho^{-1} \dot{\boldsymbol{A}}=-\mu \dot{\boldsymbol{J}}
$$

where $\boldsymbol{J}$ is the complex vector of the imposed current density, $\mu$ the magnetic permeability equal to $\mu_{0}$ in the whole domain, $\rho$ the electrical resistivity and $\omega$ the angular frequency of the magnetic field [10], [11]. The resistivity is equal to $1.710^{-8}$ $\Omega \mathrm{m}$, which is the approximate value of copper resistivity at a working temperature of $50{ }^{\circ} \mathrm{C}$ i.e. the temperature of the water-cooled inductor.

\section{THE INVERSE PROBLEM}

The problem of identifying the optimal distribution of the turn radii, assuming the two-dimensional controlled region $\Omega$ for the prescribed magnetic field, has been solved. To this end, the design variable vector $x$ was composed of ten unknown radii $R_{i}$, free to vary in the range $[65,150] \mathrm{mm}$, and by the unknown distance $d$ between the turns, free to vary in the range $[2,12] \mathrm{mm}$; altogether an eleven-dimensional search space.

The optimization problem reads as follows: given a set of frequencies $f_{k}, k=1, N$ within a prescribed range $\left(f_{L}, f_{H}\right)$, for each frequency find the family of inductor geometries that minimize the discrepancy between the highest value of the magnetic field $H_{\max }$ and the lowest value of the magnetic field $H_{\min }$ in the controlled region $\Omega$

$$
f_{1}(x)=H_{\max }(x)-H_{\min }(x)
$$

and, simultaneously, minimize the specific powerloss, $P_{i}(x)$,in the winding, i.e. the power in the most lossy turn

$$
f_{2}(x)=\max _{i}\left(P_{i}(x)\right) \text { with } i=1, \ldots, 10
$$

subject to field equation (1).

It has to be noted that the power $P_{i}$ in (3) incorporates both additional and proximity losses.
Consequently, a set of $N$ frequency-linked Pareto-optimal fronts is to be identified.

\section{The Optimization AlgorithMS}

In order to solve (2) and (3), a comparison of stochastic optimization algorithms is considered. In particular, NSGA-II, WDO, $\mu$ BiMO, MNSGA-III are used. NSGA-II is a well known genetic algorithm developed by Deb [4].

Specifically, WDO is inspired by the atmospheric motion of air parcels. In fact, wind blows in a way to equalise imbalances in the air pressure; likewise, in optimisation, the design points are moved from high-gradient to low-gradient positions. Accordingly, a swarm of $p>1$ artificial air parcels is considered. The WDO algorithm is governed by the following equation:

$$
u\left(t_{k+1}\right)=(1-\alpha) u_{i}\left(t_{k}\right)-g x_{i}+\beta\left|1-i^{-1}\right|\left(x_{o p t}-x_{i}\right)+2 u^{\perp}
$$

where the left-hand side gives the velocity of the $i$ th out of $p$ air parcels at time $t_{\mathrm{k}+1}$. The right-hand side is characterised by the following four terms:

- the inertia term, depending on velocity at time $t_{\mathrm{k}}$ and frictional coefficient $\alpha$ (conservative operator);

- the gravitational-like term $g x$, which biases the current position $x_{i}$ towards the gravity centre of the design space ('pull-in' operator);

- the pressure-gradient term (the main operator), where the index $i \geq 1$ is proportional to the pressure value at position $\mathrm{x}_{\mathrm{i}}$ while $x_{\text {opt }}$ is the position of the lowest pressure found in the previous $k-1$ iterations;

- the Coriolis-like acceleration term, due to which the velocity of the $i$ th parcel is influenced by the orthogonal velocity of another, randomly selected parcel ('information-exchange' operator).

The positive-valued constants $(\alpha, \beta, g)$ are algorithmdependent parameters. The position of the $i$ th air parcel at iteration $k+1$ is then updated as

$$
x_{i}\left(t_{k-1}\right)=x_{i}\left(t_{k}\right)+u_{i}\left(t_{k+1}\right)
$$

and the boundaries are checked to prevent any air parcel from exiting the design space. The procedure continues until the maximum number of iterations is reached.

A generalization of the algorithm, making it possible to solve a multi-objective optimisation problem with $m>1$ objective functions in conflict (M-WDO), was proposed in [5].

In turn, the $\mu$-BiMO algorithm [6] is a modification of the BiMO algorithm, which is an extension of the BBO. The BBO algorithm is based on the process of natural immigration and emigration of species between small islands in the search for more friendly habitats, as observed in nature [12]. Each solution considered is treated as a habitat or an island (design vector or individual in genetic algorithms) composed of suitability index variables (SIV, design variables), and each habitat exhibits a quality given by the habitat suitability index (HSI, objective function). The ecosystem, which is the whole set of islands or habitats, is progressively modified by means 
of two stochastic operators, i.e. migration and mutation, where migration improves the HSI of poor habitats by sharing features from good habitats (exploitation step), while mutation modifies some randomly selected SIV of a few habitats in view of a better search in the design space (exploration step).

The BBO algorithm has been widely used over the last decade as a single-objective algorithm for different applications; moreover, in the last two years, it has been extended to multi-objective optimisation problems (BiMO algorithm) [13]-[17], thanks to the concept of generalized fitness.

In $\mu \mathrm{BiMO}$, the role of small rocks in the migration of individuals is considered. As in reality, the small rocks help immigrants to colonize islands that otherwise would not be reached, with the concomitant loss of the individuals who would never reach the ground; in the proposed method the rocks have the function of not wasting habitats that otherwise would never characterize an ecosystem.

In particular, during the migration procedure, it could happen that good habitats are replaced. To recover this, the discarded habitats are stored in a vector (rock vector) that tracks the habitats.

In BiMO, when the number of islands is very small, during the processes of immigration and emigration, the generation of duplicates is a frequent event. Instead of generating new habitats randomly, they are taken from the best habitats belonging to the rock vector.

Eventually, the new Migration NSGA (MNSGA-III) algorithm, is based on the classical NSGA-II multi-objective algorithm [4] and SA-MNSGA [18], but it includes the periodical migration of a new small population (maximum $N_{p} / 2$ individuals where $N_{p}$ is the size of the initial population) that modifies the genetic heritage of the current population. It evolves like the classical NSGA-II algorithm and includes migration like SA-MNSGA [18], but the migrated population occurs before the generation step instead of after, as in SAMNSGA. This way the new individuals increase the current population and the new chromosome characteristics act in the generation of the new population. The selection step reports the population size to $N_{p}$.

\section{V.OPTIMIZATION RESULTS}

The NSGA-II method was run with 20 individuals and 100 iterations, WDO with 20 parcels and 100 iterations, $\mu$-BiMO with 8 islands and 100 iterations, MNSGA-III with 20 individuals and 50 runs. NSGA-II and WDO used 2,000 objective functions calls, $\mu$-BiMO about 800, MNSGA-III about 1,400 calls.

Due to the large number of design variables, it is quite difficult to solve this optimization problem; therefore, a further run with MNSGA-III has been performed for 100 iterations and 20 individuals (about 2,900 objective function calls).

The optimization results obtained at frequencies of $1 \mathrm{kHz}$, $10 \mathrm{kHz}$ and $100 \mathrm{kHz}$ are shown in Figs. 2, 3 and 4, respectively. In general, the best Pareto front approximation is obtained by MNSGA-III when run for 100 iterations.
In the case of $\mathrm{f}=1 \mathrm{kHz}, \mathrm{MNSGA}-\mathrm{III}$ outperforms the other methods even with 50 iterations. On the other hand, the $\mu$ BiMO method is able to find some solutions, which approximate the Pareto front, with a low computational cost. This is a typical behavior of $\mu$-BiMO, because few islands are used. Eventually, NSGA-II is able to minimize the function $\mathrm{f}_{2}$, finding a good approximation of the right part of the Pareto front for $\mathrm{f}=1 \mathrm{kHz}$.

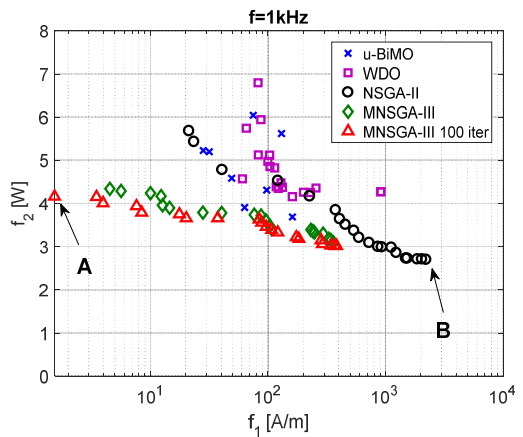

Fig. 2. Optimization results obtained for $\mathrm{f}=1 \mathrm{kHz}$.

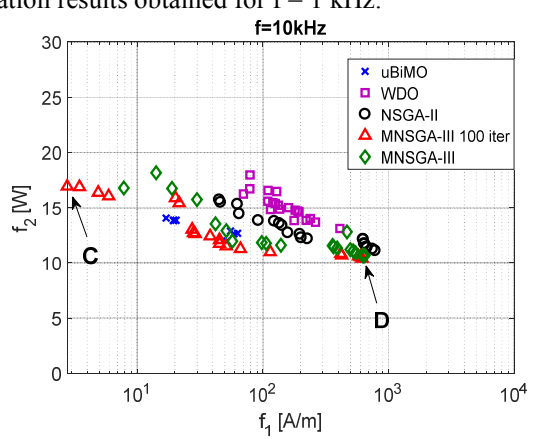

Fig. 3. Optimization results obtained for $f=10 \mathrm{kHz}$.

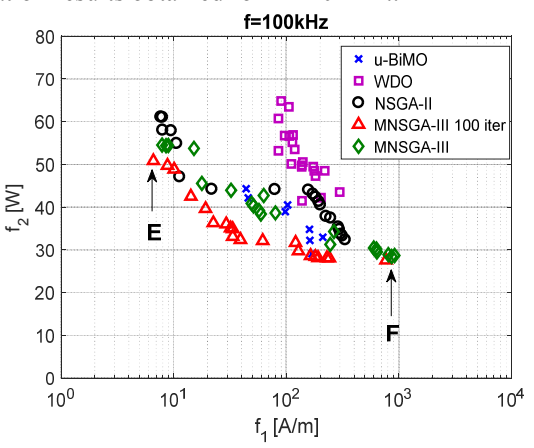

Fig. 4. Optimization results obtained for $\mathrm{f}=100 \mathrm{kHz}$.

The geometries of the solutions $\mathrm{A}$ and $\mathrm{B}, \mathrm{C}$ and $\mathrm{D}, \mathrm{E}$ and $\mathrm{F}$ obtained for $\mathrm{f}=1 \mathrm{kHz}, \mathrm{f}=10 \mathrm{kHz}$ and $\mathrm{f}=100 \mathrm{kHz}$ are shown in Figs. 5, 6 and 7, respectively. Referring to the optimal geometries $\mathrm{A}, \mathrm{C}$ and $\mathrm{E}$ in Figs. 5 to 7 , the mean value of the magnetic field is $3694 \mathrm{~A} / \mathrm{m}$ for $\mathrm{f}=1 \mathrm{kHz}, 3717 \mathrm{~A} / \mathrm{m}$ for $\mathrm{f}=10$ $\mathrm{kHz}$ and $3979 \mathrm{~A} / \mathrm{m}$ for $\mathrm{f}=100 \mathrm{kHz}$, under the same value of the supply current equal to $I_{0}=100$ Aturns.

Considering the linearity of the magnetic region (air-cored winding), the value of the magnetic field can be rescaled based on the actual current value $I$, leaving the field uniformity level unaltered. The latter remark is particularly important because the objective function $f_{l}$ attempts to reduce the highest field discrepancy in the controlled region as much as possible, regardless of the mean value of the field. The updated value of 
the field depends on the coefficient $I / I_{0}$, whereas the updated value of the losses depends on the squared coefficient. From this viewpoint, the results obtained are general.

TABLE I DESIGN VARIABLES [mm] AND OBJeCTIVE FUNCTION VALUES FOR SOLUTIONS LOCATED AT PARETO FRONT ENDS.

\begin{tabular}{c|cccccc}
\hline $\begin{array}{l}\text { f [kHz] } \\
\text { point Figs. } \\
\mathbf{2 - 4}\end{array}$ & $\mathbf{1}$ & $\mathbf{1}$ & $\mathbf{1 0}$ & $\mathbf{1 0}$ & $\mathbf{1 0 0}$ & $\mathbf{1 0 0}$ \\
\hline $\mathbf{R}_{\mathbf{1}}$ & $\mathbf{A}$ & $\mathbf{B}$ & $\mathbf{C}$ & $\mathbf{D}$ & $\mathbf{E}$ & $\mathbf{F}$ \\
$\mathbf{R}_{\mathbf{2}}$ & 130.0 & 65.0 & 134.7 & 104.6 & 116.8 & 78.87 \\
$\mathbf{R}_{\mathbf{3}}$ & 123.7 & 65.0 & 123.7 & 106.9 & 114.4 & 81.72 \\
$\mathbf{R}_{\mathbf{4}}$ & 131.3 & 65.0 & 118.5 & 66.83 & 113.6 & 75.13 \\
$\mathbf{R}_{\mathbf{5}}$ & 116.9 & 65.0 & 134.2 & 65.00 & 106.2 & 69.27 \\
$\mathbf{R}_{\mathbf{6}}$ & 116.5 & 69.9 & 110.1 & 150.0 & 93.65 & 67.15 \\
$\mathbf{R}_{\mathbf{7}}$ & 103.7 & 147.9 & 104.4 & 65.00 & 92.73 & 66.80 \\
$\mathbf{R}_{\mathbf{8}}$ & 96.66 & 65.0 & 110.3 & 65.00 & 150.0 & 67.08 \\
$\mathbf{R}_{\mathbf{9}}$ & 95.38 & 67.8 & 102.4 & 68.23 & 149.8 & 147.1 \\
$\mathbf{R}_{\mathbf{1 0}}$ & 90.91 & 133.9 & 149.9 & 65.36 & 103.2 & 81.12 \\
step $_{\mathbf{f}_{\mathbf{1}}[\mathbf{A m}}$ & 11.75 & 12.0 & 12.00 & 12.00 & 11.55 & 12.00 \\
$\mathbf{f}_{\mathbf{2}}[\mathbf{W}]$ & 1.53 & 2215 & 2.78 & 617.4 & 6.61 & 769.2 \\
\hline
\end{tabular}

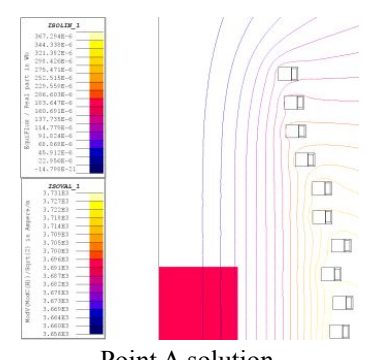

Point A solution

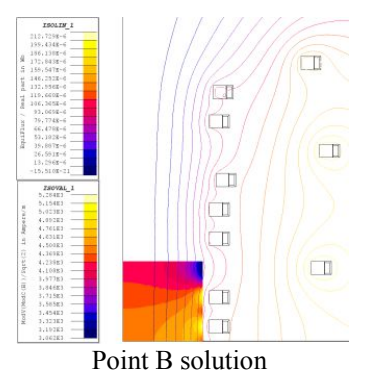

Point B solution

Fig. 5. Geometries of front-end points $\mathrm{A}$ and $\mathrm{B}, \mathrm{f}=1 \mathrm{kHz}$, in Fig. 2.
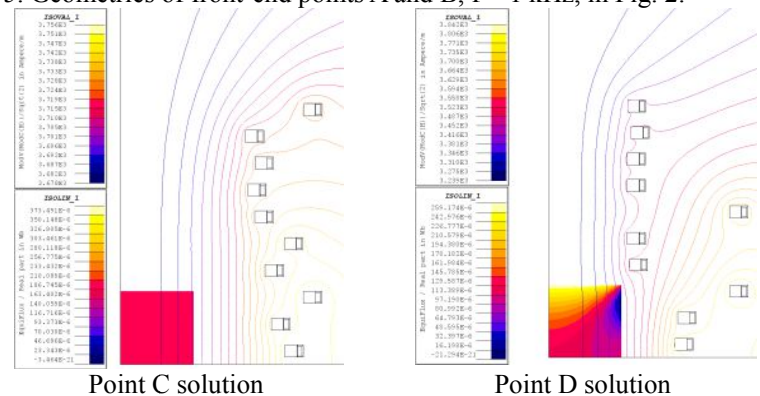

Fig. 6. Geometries of front-end points $\mathrm{C}$ and $\mathrm{D}, \mathrm{f}=10 \mathrm{kHz}$, in Fig. 3 .
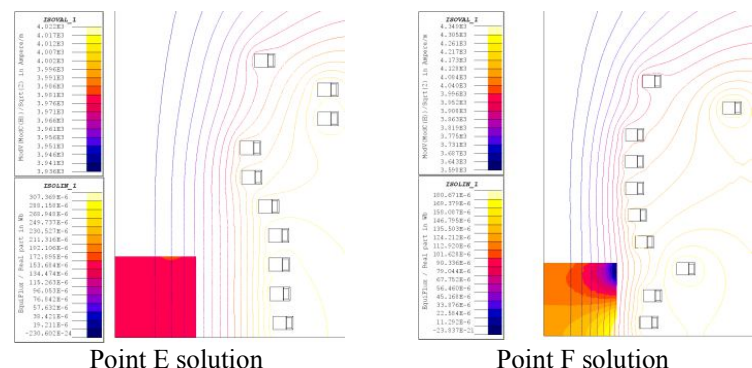

Point F solution

Fig. 7. Geometries of front-end points $\mathrm{E}$ and $\mathrm{F}, \mathrm{f}=100 \mathrm{kHz}$, in Fig. 4.

Moreover, observing the objective spaces relevant to the three frequencies, it can be also noted that the dependence of the optimal solutions on the objective function $f_{l}$ is substantial in all cases, while the higher the frequency, the more sensitive is the dependence on objective function $f_{2}$.

\section{CONCLUSION}

Despite the proposed underpinning forward problem being quite simple (time-harmonic analysis of an air-cored winding), the associated inverse problem of synthesizing a uniform field with high-energy efficiency is challenging and relevant to many industrial applications. In this paper we demonstrate that, despite the goodness of the algorithms [5-6], [13-18], the results obtained by them are not the same: this happens because the problem is tricky, hence the proposed benchmark confirms its validity.

\section{REFERENCES}

[1] “Testing Electromagnetic Analysis Methods (T.E.A.M.)." [Online]. Available: http://www.compumag.org/jsite/team.html. [11-May-2017].

[2] P. Di Barba, M. E. Mognaschi, D. A. Lowther, and J. K. Sykulski, "A Benchmark TEAM Problem for Multi-Objective Pareto Optimization of Electromagnetic Devices," IEEE Transactions on Magnetics, vol. 54, no. 3, pp. 1-4, Mar. 2018.

[3] P. Di Barba et al., "A benchmark problem of induction heating analysis," IJAEM, vol. 53, no. S1, pp. S139-S149, 2017.

[4] K. Deb, A. Pratap, S. Agarwal, and T. Meyarivan, "A fast and elitist multiobjective genetic algorithm: NSGA-II," Evolutionary Computation, IEEE Transactions on, vol. 6, no. 2, pp. 182-197, 2002.

[5] P. Di Barba, "Multi-objective wind-driven optimisation and magnet design," Electronics Letters, vol. 52, no. 14, pp. 1216-1218, Jul. 2016.

[6] M. E. Mognaschi, "Micro biogeography-inspired multi-objective optimisation for industrial electromagnetic design," Electronics Letters, vol. 53, no. 22, pp. 1458-1460, Oct. 2017.

[7] E. Sieni, P. Di Barba, and M. Forzan, "Migration NSGA: method to improve a non-elitist searching of Pareto front, with application in magnetics," Inverse Problems in Science and Engineering, vol. 24, no. 4, pp. 543-566, May 2016.

[8] "Flux electromagnetic and thermal finite element software." [Online]. Available: https://altairhyperworks.com/product/flux/Capabilities--Robust-Solver. [Accessed: 11-May-2017].

[9] "MagNet." [Online]. Available: https://www.mentor.com/products/mechanical/magnet/magnet/. [05Jun-2019].

[10] K. J. Binns, P. J. Lawrenson, and C. W. Trowbridge, The analytical and numerical solution of electric and magnetic fields. Chichester: Wiley, 1992.

[11] P. Di Barba, A. Savini, and S. Wiak, Field models in electricity and magnetism. [Dordrecht]: Springer, 2008.

[12] D. Simon, "Biogeography-Based Optimization," Evolutionary Computation, IEEE Transactions on, vol. 12, no. 6, pp. 702-713, Dec. 2008.

[13] P. Di Barba, M. E. Mognaschi, F. Dughiero, M. Forzan, and E. Sieni, "Multi-Objective Optimization of a Solenoid for MFH: A Comparison of Methods," in IECON 2018 - 44th Annual Conference of the IEEE Industrial Electronics Society, D.C., DC, USA, 2018, pp. 3336-3340.

[14] Di Barba P., F. Dughiero, M. Forzan, M. E. Mognaschi, and Sieni E., "New solutions to a multi-objective benchmark problem of induction heating: an application of computational biogeography and evolutionary algorithms," Archives of Electrical Engineering, vol. 67, no. 1, pp. 139-149, 2018.

[15] P. Di Barba, M. E. Mognaschi, and A. Krawczyk, "The biogeographyinspired optimization for the design of coils for nerve stimulation," 2017, 17th IEEE International Conference on Smart Technologies, EUROCON 2017 - Conference Proceedings, pp. 542-545.

[16] P. Di Barba, M. E. Mognaschi, P. Venini, and S. Wiak, "Biogeography-inspired multiobjective optimization for helping MEMS synthesis," Archives of Electrical Engineering, vol. 66, no. 3, Jan. 2017.

[17] P. Di Barba, F. Dughiero, M. E. Mognaschi, A. Savini, and S. Wiak, "Biogeography-Inspired Multiobjective Optimization and MEMS Design," IEEE Transactions on Magnetics, vol. 52, no. 3, pp. 1-4, Mar. 2016.

[18] P. Di Barba, F. Dughiero, M. Forzan, and E. Sieni, "SELF-adaptive Migration-NSGA algorithm: An application in uncertainty-tolerant magnetic field synthesis for MFH inductor design," IJAEM, vol. 56, pp. 17-32, Feb. 2018. 